PROCEEDINGS OF THE

AMERICAN MATHEMATICAL SOCIETY

Volume 129, Number 3, Pages 667-669

S 0002-9939(00)05626-4

Article electronically published on September 19, 2000

\title{
TIGHT CLOSURE COMMUTES WITH LOCALIZATION IN BINOMIAL RINGS
}

\author{
KAREN E. SMITH
}

(Communicated by Wolmer V. Vasconcelos)

\begin{abstract}
It is proved that tight closure commutes with localization in any domain which has a module finite extension in which tight closure is known to commute with localization. It follows that tight closure commutes with localization in binomial rings, in particular in semigroup or toric rings.
\end{abstract}

Tight closure, introduced in [HH1, is a closure operation performed on ideals in a commutative, Noetherian ring containing a field. Although it has many important applications to commutative algebra and related areas, some basic questions about tight closure remain open. One of the biggest such open problems is whether or not tight closure commutes with localization. For an exposition of tight closure, including the localization problem, see $\mathrm{Hu}$.

The purpose of this paper is give a simple proof that tight closure commutes with localization for a class of rings that includes semi-group rings and, slightly more generally, the so-called "binomial" rings, that is, those that are a quotient of a finitely generated algebra by an ideal generated by binomials. Although this is a modest class of rings, the result here includes coordinate rings of all affine toric varieties, and is general enough to substantially improve previous results of a number a mathematicians, including Moty Katzman, Will Traves, Keith Pardue and Karen Chandler, Irena Swanson and myself.

Throughout this note, $R$ denotes a Noetherian commutative ring of prime characteristic.

We say that tight closure commutes with localization in $R$ if, for all ideals $I$ in $R$ and all multiplicative systems $U$ in $R, I^{*} R\left[U^{-1}\right]=\left(I R\left[U^{-1}\right]\right)^{*}$.

The main result is the following:

Theorem. Let $R$ be a ring with the following property: for each minimal prime $P$ of $R$, the quotient $R / P$ has an integral extension domain in which tight closure commutes with localization. Then tight closure commutes with localization in $R$.

Lemma 1. If tight closure commutes with localization in $R / P$ for each minimal prime $P$ of $R$, then tight closure commutes with localization in $R$.

Received by the editors January 11, 1999 and, in revised form, May 15, 1999.

1991 Mathematics Subject Classification. Primary 13A35.

Key words and phrases. Tight closure, localization, binomial ideals, toric rings, semigroup rings.

The author was supported by the National Science Foundation and the Alfred P. Sloan Foundation.

(C)2000 American Mathematical Society 
Proof. (See also [AHH1, 3.8].) It follows immediately from the definitions that $I^{*} R\left[U^{-1}\right] \subset\left(I R\left[U^{-1}\right]\right)^{*}$ in general. To prove the reverse inclusion, let $\frac{z}{1}$ be in $\left(\operatorname{IR}\left[U^{-1}\right]\right)^{*}$. Then modulo each minimal prime of $R$ disjoint from $U$, the image of $\frac{z}{1}$ is in $\left(I R / P\left[U^{-1}\right]\right)^{*}=(I R / P)^{*} R / P\left[U^{-1}\right]$. For each minimal prime $P_{i}$ disjoint from $U$, there is some $u_{i}$ in $U$ such that $u_{i} z$ is in $\left(I R / P_{i}\right)^{*}$. By setting $u=\prod u_{i}$, we see that $u z \in(I R / P)^{*}$ for all minimal primes $P$ of $R$ disjoint from $U$. On the other hand, for each minimal prime $Q$ that meets $U$, pick any any element in $Q \cap U$ and let $v$ be their product. Now $u v z \in(I R / P)^{*}$ for every minimal prime $P$ of $R$, which means that $u v z \in I^{*}$ in $R$. But because $u u v \in U$, we conclude that $\frac{z}{1} \in I^{*} R\left[U^{-1}\right]$ and tight closure commutes with localization in $R$.

Lemma 2. Let $R$ be a domain that has a finite extension domain $S$ in which tight closure commutes with localization. Then tight closure commutes with localization in $R$.

Proof. Let $z \in R$ and let $I \subset R$. If $\frac{z}{1} \in\left(I R\left[U^{-1}\right]\right)^{*}$, then the equations $c\left(\frac{z}{1}\right)^{q} \in$ $I^{[q]} R\left[U^{-1}\right]$ hold also after expansion to $S\left[U^{-1}\right]$, so that $\frac{z}{1}$ is in $\left(I S\left[U^{-1}\right]\right)^{*}$. Since tight closure commutes with localization in $S$, we have $\frac{z}{1}$ is in $(I S)^{*} S\left[U^{-1}\right]$. This means that there exists a $u \in U$ such that $u z \in(I S)^{*}$. But in general it is easy to see that $(I S)^{*} \cap R \subset I^{*}$ for any ideal $I$ of $R$ and any integral extension domain $S$ of $R$, so $u z \in I^{*}$. (Indeed, by tensoring with the fraction field of $R$ and then clearing denominators, one can find an $R$-linear map $S \stackrel{\phi}{\rightarrow} R$ which sends a fixed non-zero $c$ in $S$ to some non-zero element $d$ of $R$. Then we can apply $\phi$ to the equations expressing $c(z u)^{q} \in I^{[q]} S$ to get equations expressing $d(u z)^{q} \in I^{[q]}$ in $R$. This implies that $\frac{z}{1} \in I^{*} R\left[U^{-1}\right]$ and the proof is complete.)

Proof of the Theorem. By the first lemma, it suffices to consider the case where $R$ is a domain. The proof is complete by the second lemma, since every integral extension is a directed limit of module finite extensions.

Corollary. If $R$ has the property that modulo each minimal prime, the ring $R / P$ has an integral extension that is F-regular, then tight closure commutes with localization in $R$.

Proof. By definition, a ring is F-regular if all ideals are tightly closed both in the ring and in all its localizations. Thus tight closure trivially commutes with localization in an F-regular ring, and the corollary follows immediately from the Theorem.

Corollary. Let $R$ be a ring of the form $k\left[x_{1}, \ldots, x_{n}\right] / J$ where $J$ is generated by binomials in the $x_{i}$ 's. (A binomial is any polynomial of the form $\lambda x_{1}^{a_{1}} \ldots x_{n}^{a_{n}}-$ $\mu x_{1}^{b_{1}} \ldots x_{n}^{b_{n}}$, where $\lambda$ and $\mu$ are elements of $k$. In particular, every monomial is a binomial in this sense.) Then tight closure commutes with localization in $R$.

Proof. There is no loss of generality in assuming the field is algebraically closed, by an argument similar to the one used to prove Lemma 2 (we can apply Lemma 2 directly if $\bar{k} \otimes_{k} R$ is a domain where $\bar{k}$ is the algebraic closure of $k$ ). In this case, the minimal primes of $J$ are themselves binomial ideals [ES], so by Lemma 1, we may assume that $R$ is a domain. Now, a ring which is the quotient of a polynomial ring by a prime binomial ideal is isomorphic to a subring of a polynomial ring $k\left[t_{1}, t_{2}, \ldots, t_{d}\right]$ generated by monomials in the $t_{i}$ 's, so we assume $R$ is such a monomial algebra [ES, 2.3]. The normalization $\tilde{R}$ of such a monomial algebra is again a monomial algebra. On the other hand, a normal monomial algebra is isomorphic to a direct 
summand of the polynomial overrring $k\left[t_{1}, \ldots, t_{d}\right]$ (see, e.g., [BH, Exercise 6.1.10]). Since a direct summand of a regular ring (or even F-regular ring) is F-regular, we see that $\tilde{R}$ is F-regular, so by the Corollary above, tight closure commutes with localization in $R$.

Special cases of this corollary, including the case where $J$ is generated by monomials, had been observed using different methods by Will Traves, Moty Katzman, Keith Pardue and Karen Chandler, and Irena Swanson and myself. Katzman's work [K] includes an interesting approach to the localization problem using Gröbner basis (suggested by Craig Huneke) in the special case of binomial ideals. Aldo Conca [C], and Karen Chandler and Keith Pardue also made progress towards this Gröbner basis approach in the binomial ideal case. In [SS], a different approach to the localization problem is considered, in which the growth of the primary components of Frobenius powers is analyzed; the problem is solved for monomial ideals in monomial rings. In T, the monomial case is solved using differential operators. The thesis of Doug McCulloch $[\mathrm{Mc}]$ does not directly address the localization problem, but contains a systematic study of tight closure in binomial rings.

\section{REFERENCES}

[AHH1] Aberbach, I., Hochster, M. and Huneke, C., Localization of tight closure and modules of finite phantom projective dimension, J. Reine Angew. 434 (1993), 67-114. MR 94h:13005

[BH] Bruns, W. and Herzog, J., Cohen-Macaulay Rings, vol. 39, Cambridge Studies in Advanced Mathematics, 1993. MR 95h:13020

[C] Conca, A., Hilbert-Kunz function of monomial ideals and binomial hypersurfaces, Manuscripta Math. 90 (1996), 287-300. MR 97g:13006

[ES] Eisenbud, D. and Sturmfels, B., Binomial ideals, Duke 84 (1996), 1-45. |MR 97d:13031

[HH1] Hochster, M. and Huneke, C., Tight closure, invariant theory, and the Briancon-Skoda theorem, JAMS 3 (1990), 31-116. MR 91g:13010

$[\mathrm{Hu}]$ Huneke, C., Tight closure and its applications, vol. 88, CBMS Conference Series, American Mathematical Society, Providence, RI, 1996. MR 96m:13001

[K] Katzman, Mordechai, The complexity of Frobenius powers of ideals, J. Algebra 203 (1998), 211-225. MR 99e:13003

[Mc] McCulloch, D., Tight closure and base change, thesis, University of Michigan (1997).

[SS] Smith, K.E. and Swanson, I., Linear growth of primary components of monomial ideals, Communications in Algebra 25 (1997), 3071-3079.

[T] Traves, W., Differential operators on monomial rings, Jour. Pure and Applied Algebra 136 (1999), 183-197. CMP 99:09

Department of Mathematics, University of Michigan, Ann Arbor, Michigan 48109

E-mail address: kesmith@math.1sa.umich.edu 